\title{
Supporting Information: A Rational Approach to Minimal High-Resolution Cross-Reactive Arrays
}

\author{
Eric Green ${ }^{a}$, Mark J. Olah ${ }^{b}$, Tatiana Abramova ${ }^{a}$, Lance R. Williams ${ }^{b}$, \\ Darko Stefanovic $^{b}$, Tilla Worgall ${ }^{a}$, and Milan N. Stojanovic ${ }^{a}$ \\ ${ }^{a}$ Division of Clinical Pharmacology and Experimental Therapeutics, \\ Columbia University, New York, NY 10032 \\ ${ }^{b}$ Department of Computer Science, University of New Mexico, Albuquerque, NM 87131
}

May 12, 2006 
Table 1. Error rates of several projections of the data when used as training data for a $K$-Nearest-Neighbors classifier, with $k=3$ and ties broken by the closest neighbor.

\begin{tabular}{lr}
\hline Projection & Error Rate \\
\hline All 7 Sensors & $5.51 \% \pm 2.74 \%$ \\
Optimal 6-sensor Array & $3.87 \% \pm 2.26 \%$ \\
Optimized Curve Separation 2D & $27.76 \% \pm 5.62 \%$ \\
Linear PCA 3D & $8.40 \% \pm 3.40 \%$ \\
Linear PCA 2D & $19.93 \% \pm 4.99 \%$ \\
Quadratic PCA 3D & $26.52 \% \pm 5.54 \%$ \\
Quadratic PCA 2D & $36.82 \% \pm 6.07 \%$ \\
\hline
\end{tabular}

\section{Visualization of Results}

In the paper, Figure 4, presents a $2 \mathrm{D}$ projection which was obtained via a new projection persuit algorithm. The algorithm represents each class (steroid) as a segmented curve in the seven dimensional space of sensors. The segmented curve for a class is obtained by connecting the mean responses for each concentration value measured for that class. Given this representation, the algorithm attempts to find the $2 \mathrm{D}$ projection which maximizes the minimum distance between the segmented curves. This is done by formulation of the problem as an optimization proceedure, and applying the Downhill Simplex Method for function maximization. Since the objective function is not convex, there exist many local maximums. Therefore, the algorithm is run multiple times on randomized starting positions in order to obtain a local maximum close to the global maximum.

The purpose of the 2D projection is to provide a useful visual impression of the data. While the projection gives accurate information about the relationship of the data within a specific 2D supspace, this relationsip is not related to the relationship learned by the KNN-classifier on the optimal sensor set in 6D. In fact, training a classifier using this optimized 2D projection leads to poor accuracy as shown in Table 1. This is an indication that the projection into 2D has removed critical features of the classes needed for accurate classification. This is not surprising, as other dimensionality reduction methods such as PCA and Kernel PCA with non-linear kernels also have poor performance on our data set when the dimensionality is reduced to $2 \mathrm{D}$ or $3 \mathrm{D}$.

Since all of the 2D and 3D representations of the data examined have high error rates when used to train a classifier, the relationsips they represent cannot provide any useful insight into the mechanisms learned by the optimal classifier. While there may be other $2 \mathrm{D}$ or $3 \mathrm{D}$ projections which can train classifiers which achieve slightly better error rates, it is very unlikely that any of them will come close to the error rates obtained by the optimal classifier.

\section{Confusion Matrices}

When examining a classifier, it is easy to generate estimations of its overall classification accuracy. However, the overall accuracy does not give any information concerning the specific types of errors that a classifier is making. Knowing which classes are often confused provides usefull feedback that helps to identify weak points in the sensor coverage, and allows more detailed comparison of different sensor subsets. One method to investigate the specific errors of a classifier, is to generate a confusion matrix. Given a classifier, and an estimator (in our case the Leave-One-Out estimator), the estimator will 
make a series of classification attempts, and report back an estimate of the error based on how many misclassification occurred. A confusion matrix can be generated by recording the action of the classifier on each of these attempted classifications. Each row in the matrix represents the true class of the sample, and each column represents the class assigned to that sample. Thus if row 5, column 2 has value 3, it means that three samples with true class $\mathbf{5}$ were missclassified as $\mathbf{2}$. Therefore, all counts on the diagonal represent correct classifications, while all counts off the diagonal represent errors.

We generated confusion matrices for the top five ranked sensor arrays, so that we could get better information about the strengths and weaknesses of each of the arrays. Also provided are the number of missclassifications, and the $95 \%$ confidence intervals on the error rates as estimated by the LOO method.

\section{List of Confusion Matrices}

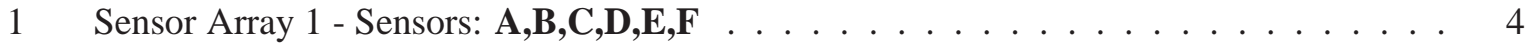

2 Sensor Array 2 - Sensors: $\mathbf{A}, \mathbf{B}, \mathbf{C}, \mathbf{D}, \mathbf{F} \ldots \ldots \ldots \ldots$

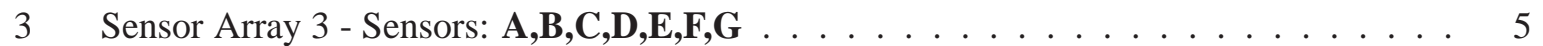

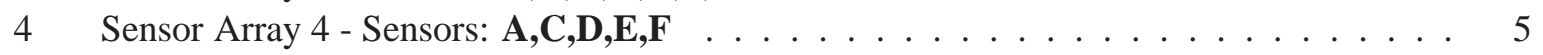

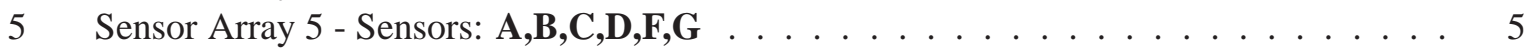


Confusion Matrix 1. Sensor Array 1 - Sensors: A,B,C,D,E,F

Assigned Class

\begin{tabular}{c|c|c|c|c|c|c|c|c|} 
& \multicolumn{7}{|c}{ Assigned Class } \\
& $\mathbf{1}$ & $\mathbf{2}$ & $\mathbf{3}$ & $\mathbf{4}$ & $\mathbf{5}$ & $\mathbf{6}$ & $\mathbf{7}$ & $\mathbf{8}$ \\
\hline $\mathbf{1}$ & $\mathbf{5 2}$ & 0 & 0 & $\mathbf{1}$ & 0 & 0 & $\mathbf{1}$ & 0 \\
\hline $\mathbf{2}$ & 0 & 42 & 0 & 0 & 0 & 0 & 0 & 0 \\
\hline $\mathbf{5}$ & 0 & 0 & 29 & 0 & 0 & 0 & $\mathbf{1}$ & 0 \\
\hline $\mathbf{5}$ & $\mathbf{1}$ & 0 & 0 & 11 & 0 & 0 & 0 & 0 \\
\hline $\mathbf{5}$ & 0 & 0 & 0 & 0 & 17 & 0 & 0 & $\mathbf{1}$ \\
\hline $\mathbf{6}$ & 0 & $\mathbf{1}$ & 0 & 0 & 0 & 35 & 0 & 0 \\
\hline $\mathbf{7}$ & $\mathbf{2}$ & 0 & 0 & 0 & 0 & 0 & 16 & 0 \\
\hline $\mathbf{8}$ & 0 & 0 & 0 & 0 & 0 & 0 & 0 & 30 \\
\hline
\end{tabular}

Total Errors:

Error Rate (95\% conf.):

$3.87 \% \pm \mathbf{2 . 2 6} \%$

Confusion Matrix 2. Sensor Array 2 - Sensors: A,B,C,D,F

Assigned Class

\begin{tabular}{c|c|c|c|c|c|c|c|c|} 
& \multicolumn{7}{|c}{ Assigned Class } \\
& $\mathbf{1}$ & $\mathbf{2}$ & $\mathbf{3}$ & $\mathbf{4}$ & $\mathbf{5}$ & $\mathbf{6}$ & $\mathbf{7}$ & $\mathbf{8}$ \\
\hline $\mathbf{1}$ & $\mathbf{5 2}$ & 0 & 0 & $\mathbf{1}$ & 0 & 0 & $\mathbf{1}$ & 0 \\
\hline $\mathbf{2}$ & 0 & 42 & 0 & 0 & 0 & 0 & 0 & 0 \\
\hline $\mathbf{3}$ & 0 & 0 & 29 & 0 & 0 & 0 & $\mathbf{1}$ & 0 \\
\hline $\mathbf{4}$ & $\mathbf{1}$ & 0 & 0 & $\mathbf{1 0}$ & 0 & 0 & $\mathbf{1}$ & 0 \\
\hline $\mathbf{5}$ & 0 & 0 & 0 & 0 & 17 & 0 & 0 & $\mathbf{1}$ \\
\hline $\mathbf{6}$ & 0 & $\mathbf{2}$ & 0 & 0 & 0 & 34 & 0 & 0 \\
\hline $\mathbf{7}$ & $\mathbf{2}$ & 0 & 0 & 0 & 0 & 0 & 16 & 0 \\
\hline $\mathbf{8}$ & 0 & 0 & 0 & 0 & 0 & 0 & 0 & 30 \\
\hline
\end{tabular}

Total Errors:

Error Rate (95\% conf.):

$\mathbf{4 . 6 9} \% \pm \mathbf{2 . 5 2} \%$ 
Confusion Matrix 3. Sensor Array 3 - Sensors: A,B,C,D,E,F,G

\begin{tabular}{c|c|c|c|c|c|c|c|c|} 
& \multicolumn{7}{|c}{ Assigned Class } \\
& $\mathbf{1}$ & $\mathbf{2}$ & $\mathbf{3}$ & $\mathbf{4}$ & $\mathbf{5}$ & $\mathbf{6}$ & $\mathbf{7}$ & $\mathbf{8}$ \\
\hline $\mathbf{1}$ & 53 & 0 & 0 & $\mathbf{1}$ & 0 & 0 & 0 & 0 \\
\hline $\mathbf{2}$ & 0 & 42 & 0 & 0 & 0 & 0 & 0 & 0 \\
\hline $\mathbf{3}$ & 0 & $\mathbf{1}$ & 28 & 0 & 0 & 0 & $\mathbf{1}$ & 0 \\
\hline $\mathbf{\Xi}$ & $\mathbf{1}$ & 0 & 0 & $\mathbf{1 0}$ & 0 & 0 & $\mathbf{1}$ & 0 \\
\hline $\mathbf{5}$ & 0 & 0 & 0 & 0 & 16 & 0 & 0 & $\mathbf{2}$ \\
\hline $\mathbf{6}$ & 0 & $\mathbf{2}$ & 0 & 0 & 0 & 34 & 0 & 0 \\
\hline $\mathbf{7}$ & $\mathbf{3}$ & 0 & 0 & 0 & 0 & 0 & 15 & 0 \\
\hline $\mathbf{8}$ & 0 & 0 & 0 & 0 & 0 & 0 & 0 & 30 \\
\hline
\end{tabular}

Total Errors:

Error Rate (95\% conf.): $\quad \mathbf{5 . 5 1} \% \pm \mathbf{2 . 7 4} \%$

Confusion Matrix 4. Sensor Array 4 - Sensors: A,C,D,E,F

\begin{tabular}{c|c|c|c|c|c|c|c|c|} 
& \multicolumn{10}{|c}{ Assigned Class } \\
& $\mathbf{1}$ & $\mathbf{2}$ & $\mathbf{3}$ & $\mathbf{4}$ & $\mathbf{5}$ & $\mathbf{6}$ & $\mathbf{7}$ & $\mathbf{8}$ \\
\hline $\mathbf{1}$ & $\mathbf{5 3}$ & 0 & 0 & 0 & 0 & 0 & $\mathbf{1}$ & 0 \\
\hline $\mathbf{2}$ & 0 & 41 & 0 & 0 & 0 & $\mathbf{1}$ & 0 & 0 \\
\hline $\mathbf{3}$ & 0 & $\mathbf{1}$ & 28 & 0 & 0 & 0 & $\mathbf{1}$ & 0 \\
\hline $\mathbf{6}$ & 0 & 0 & 0 & 12 & 0 & 0 & 0 & 0 \\
\hline $\mathbf{5}$ & 0 & 0 & 0 & 0 & 16 & 0 & 0 & $\mathbf{2}$ \\
\hline $\mathbf{6}$ & 0 & $\mathbf{4}$ & 0 & 0 & 0 & 32 & 0 & 0 \\
\hline $\mathbf{7}$ & $\mathbf{2}$ & 0 & 0 & 0 & 0 & 0 & 16 & 0 \\
\hline $\mathbf{8}$ & 0 & 0 & 0 & 0 & 0 & 0 & 0 & 30 \\
\hline
\end{tabular}

Total Errors:

Error Rate (95\% conf.): $\quad \mathbf{5 . 5 1} \% \pm \mathbf{2 . 7 4 \%}$

Confusion Matrix 5. Sensor Array 5 - Sensors: A,B,C,D,F,G

Assigned Class

\begin{tabular}{c|c|c|c|c|c|c|c|c|} 
& \multicolumn{10}{|c}{ Assigned Class } \\
\hline & $\mathbf{1}$ & $\mathbf{2}$ & $\mathbf{3}$ & $\mathbf{4}$ & $\mathbf{5}$ & $\mathbf{6}$ & $\mathbf{7}$ & $\mathbf{8}$ \\
\hline $\mathbf{1}$ & $\mathbf{5 3}$ & 0 & $\mathbf{1}$ & 0 & 0 & 0 & 0 & 0 \\
\hline $\mathbf{2}$ & 0 & $\mathbf{4 2}$ & 0 & 0 & 0 & 0 & 0 & 0 \\
\hline $\mathbf{3}$ & 0 & $\mathbf{1}$ & 28 & 0 & 0 & 0 & $\mathbf{1}$ & 0 \\
\hline $\mathbf{4}$ & 0 & 0 & 0 & $\mathbf{1 0}$ & 0 & 0 & $\mathbf{2}$ & 0 \\
\hline $\mathbf{5}$ & 0 & 0 & 0 & 0 & 16 & 0 & 0 & $\mathbf{2}$ \\
\hline $\mathbf{6}$ & 0 & $\mathbf{3}$ & 0 & 0 & 0 & 33 & 0 & 0 \\
\hline $\mathbf{7}$ & $\mathbf{3}$ & 0 & 0 & 0 & 0 & 0 & $\mathbf{1 5}$ & 0 \\
\hline $\mathbf{8}$ & 0 & 0 & 0 & 0 & 0 & 0 & 0 & 30 \\
\hline
\end{tabular}

Total Errors:

$13 / 240$

Error Rate (95\% conf.): $\quad \mathbf{5 . 9 3} \% \pm \mathbf{2 . 8 5} \%$ 


\section{Response of sensors A, B, C, and G:}
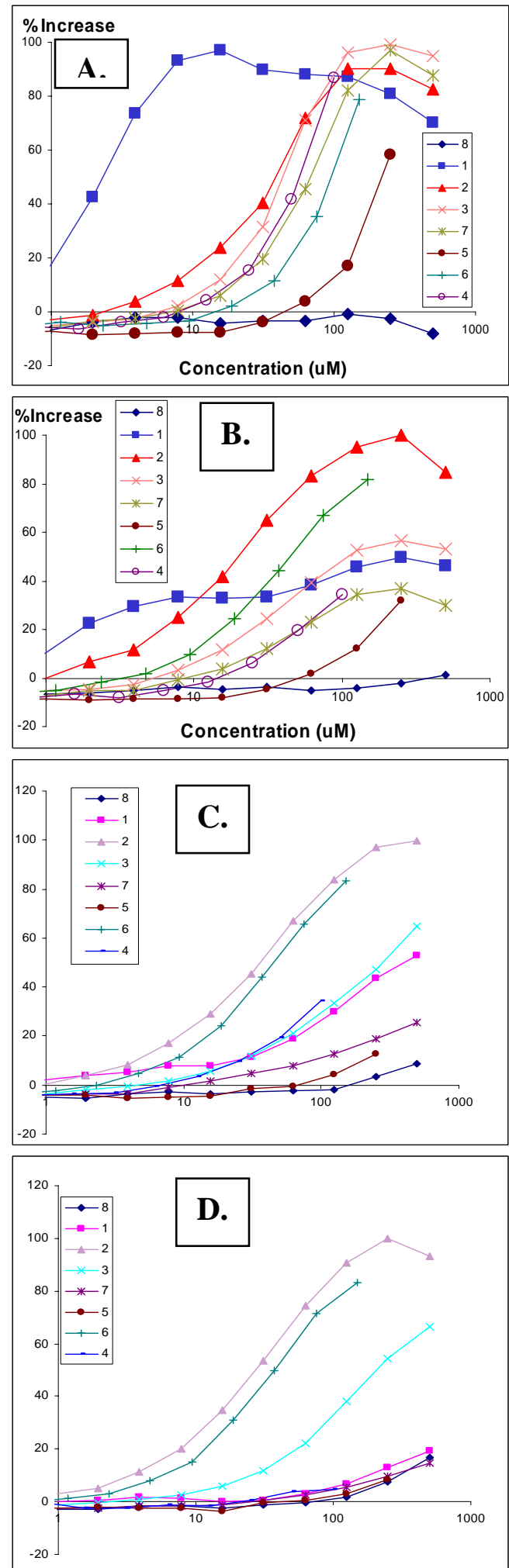

S-6 


\section{Response of sensors E, F, and G:}
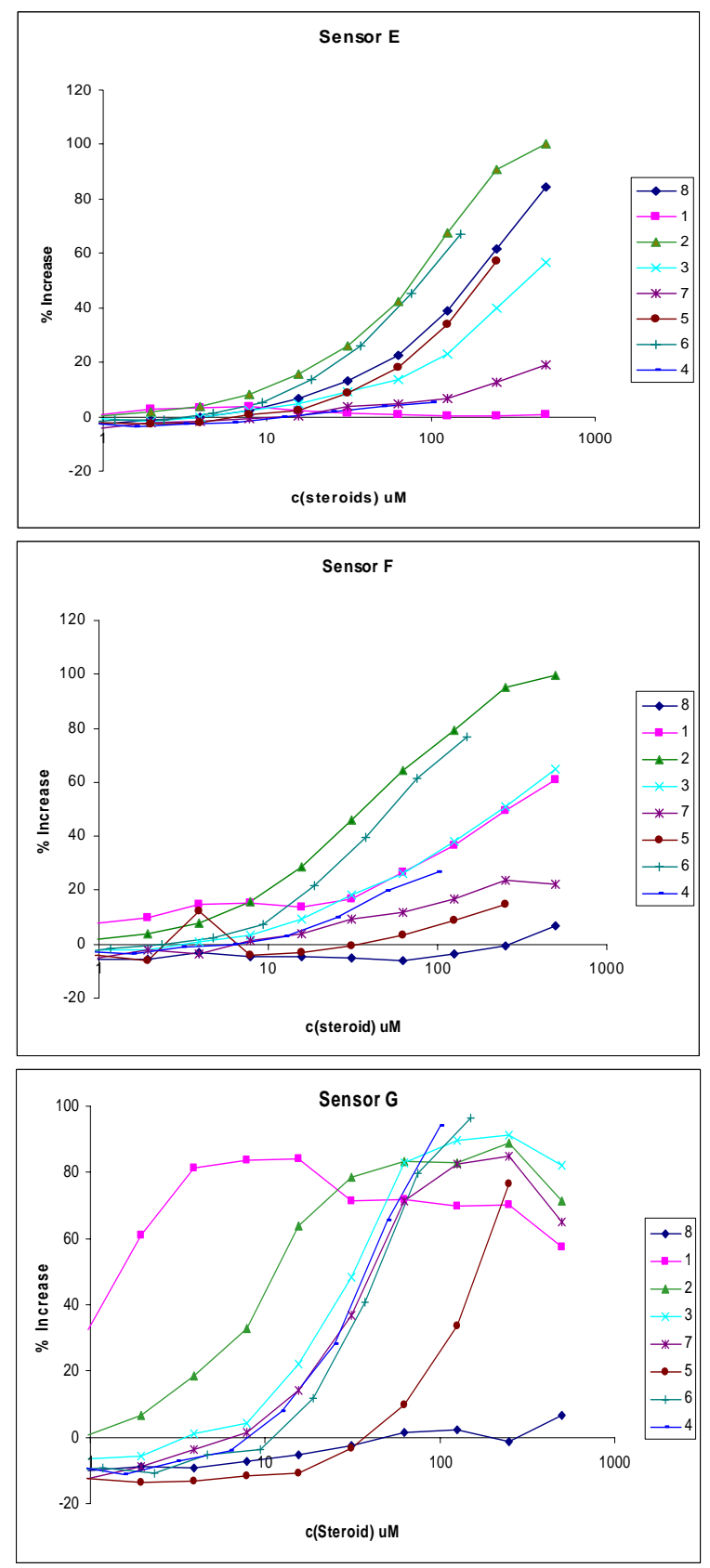